\title{
IMPROVING FAULT ISOLABILITY PROPERTIES BY STRUCTURAL ANALYSIS \\ OF FAULTY BEHAVIOR MODELS: APPLICATION TO THE DAMADICS BENCHMARK PROBLEM
}

\author{
Erik Frisk *, Dilek Düştegör ${ }^{* *}$, \\ Mattias Krysander*, and Vincent Cocquempot ${ }^{* *}$ \\ * Dept. of Electrical Engineering \\ Linköping University, SE-584 31 Linköping Sweden \\ \{frisk, matkr\}@isy.liu.se \\ ** Université des Sciences et Technologies de Lille \\ LAIL, UPRESA CNRS 8021, Bât. P2 \\ 59655 Villeneuve d'Ascq, Cedex, France \\ \{dilek.dustegor, vincent. cocquempot\}@univ-lille1.fr
}

\begin{abstract}
Structural analysis is a powerful tool for early determination of detectability/isolability possibilities. It is shown how different levels of knowledge about faults can be incorporated in a structural fault-isolability analysis and how they result in different isolability properties. The results are evaluated on the DAMADICS valve benchmark model. It is also shown how to determine which faults in the benchmark that need further modeling to get desired isolability properties of the diagnosis system.
\end{abstract}

Keywords: diagnosis, structural analysis, fault isolation, consistency relations

\section{INTRODUCTION}

Structural analysis is a powerful tool for early determination of detectability/isolability possibilities. This is important both to evaluate if the number and placement of sensors is adequate in order to meet diagnosis specifications.

Even though the structural information is very coarse, useful insights can be gained by analyzing the structure. This is also one of the strengths, since useful information can efficiently be obtained early in the development process before much work has been spent on obtaining detailed analytical models.

Structural analysis for FDI has been extensively studied, for example in (Staroswiecki et al., 2000; Pulido and Alonso, 2000; Krysander and Nyberg, 2002b). Furthermore, structural analysis has also proved useful in sensor-placement for FDI (TravéMassuyès et al., 2001) and reconfigurability analysis for fault tolerant control (Gehin et al., 2000). Here, the focus will be on fault isolability analysis.

Obtaining accurate models is expensive and difficult and this is especially true when models describing faulty behavior of the plant is considered. To obtain such models, experimental data from a faulty process or deep engineering knowledge and experience of process components are needed, neither of which may be present early in the development process. Therefore it is interesting to analyze the level of knowledge about specific faults that are needed to meet diagnosis requirements. Three levels of fault knowledge can be stated as:

(1) A basic level of knowledge is to only specify which component the fault influences. No specific information on how and in which constraints the fault influences the process is included. A component is regarded as a subset of the model equations. 
(2) A second level of fault knowledge is when it is specified which equation or equations within a component description that is subjected to fault influences, i.e. which model equations that are (possibly) not valid in case of a fault.

(3) A third level is when additional information about the fault is available and a model for the fault signal is available. This could for example be that the fault size is slowly varying and can be assumed constant, or that the fault size is highly correlated with some other signals in the model.

To exemplify the above, consider a tube that is connected to a pipe and where we can have a leakage due to a loose connection between the two components. Basic knowledge is that a leak influences the equations describing the flow through the system. With a more detailed model, we know that a leakage results in an additional flow out of the system. Thirdly, it is possible that the effective area of the leakage is dependent on the pressure in the system due to elasticity in the plastic tubing.

In summary, it is valuable to get information of what level of knowledge about faults that is necessary to obtain sufficient fault isolability properties of the diagnosis system. Structural analysis is one tool to get such information.

\section{STRUCTURAL ANALYSIS AND FAULT DETECTABILITY/ISOLABILITY}

Structural analysis is concerned with properties of the system structure. Structural information here means which variables that appear in which equations/constraints. Now it will be briefly outlined how an analysis of the structural model can provide information on fault isolability properties of the model.

The types of variables in a structural model can in a diagnosis context be divided into

(1) known variables, typically measurements and controller outputs

(2) unknown variables, typically internal states and unknown inputs that should not influence the residual

(3) faults to be detected

The model is here represented by an incidence matrix which is a matrix where each row corresponds to an equation and each column to a variable. An $X$ in position $(i, j)$ indicates that variable $j$ appears in equation $i$. Consider the small static model

$$
\begin{array}{r}
g_{1}\left(x_{1}, x_{2}, u\right)=0 \\
g_{2}\left(x_{2}, y, f\right)=0
\end{array}
$$

consisting of two equations, two unknown variables $x_{1}$ and $x_{2}$, two known variables $y, u$, and one fault $f$. The incidence matrix representation is then

\begin{tabular}{c|cc|cc|c} 
& $x_{1}$ & $x_{2}$ & $y$ & $u$ & $f$ \\
\hline$g_{1}$ & $X$ & $X$ & & $X$ & \\
$g_{2}$ & & $X$ & $X$ & & $X$
\end{tabular}

Now that representation of structural models has been introduced, fault isolability analysis of such models can be approached. The basis for diagnosis is redundancy. In consistency based diagnosis systems a crucial step is conflict recognition. Here conflicts are recognized by using precomputed consistency relations ${ }^{1}$ where a consistency relation is a static or dynamic constraint on the time evolution of known variables of the process. A consistency relation can be formally defined as

Definition 1. (Consistency relation). Let $x$ and $y$ be unknown and known variables respectively. Then, a scalar equation $c(y)=0$ is a consistency relation for a set of equations $H(x, y)$ if and only if for all $y$ it holds that

$$
\exists x . H(x, y)=0 \Rightarrow c(y)=0
$$

and there is no proper subset of $H$ that has property (1).

This means that relation $c(y) \neq 0$ is a test on inconsistency of equations $H(x, y)$ with measured data. The task of the diagnosis system designer is then to find a suitable set of such relations where different subsets of relations are invalidated by different subsets of faults. In this way, fault isolation can be achieved. This is often referred to as structured residuals.

Thus, to evaluate possible isolability properties of the model, fault sensitivity of all possible consistency relations need to be studied. Now a method is presented for performing such analysis based on the incidence matrix representation of the model. To proceed, a definition from (Krysander and Nyberg, $2002 \mathrm{~b}$ ) is needed.

Definition 2. (Minimal Structurally Singular, MSS). A finite set of equations $E$ is structurally singular with respect to variables $X$ if the number of equations is larger than the number of variables that appear in the equations, i.e. the set of equations is over determined with respect to $X$. The set $E$ is a minimal structurally singular set if none of its proper subsets are structurally singular.

An MSS should here then be regarded as a set of equations with which it is possible to derive a consistency relation. But also, due to the minimality property, all equations must be used when deriving the consistency relation. With this definition it is possible to state (under some technical model assumptions not included here):

Theorem 1. Let $\left\{E_{i}\right\}$ be the set of MSS:s. Then there exists a consistency relation for a set of equations $H$ if and only if

$$
H=\cup_{i} E_{i}
$$

PROOF. See (Krysander and Nyberg, 2002a).

1 Consistency relations are often also called Analytical Redundancy Relations (ARR). 
This result implies that the isolability properties of a system can be determined by only considering the fault sensitivities of the MSS:s. An algorithm not described further here, to compute all MSS sets and their respective fault sensitivity based on an incidence matrix can be found in (Krysander and Nyberg, 2002a).

After the set of MSS:s has been determined, isolability properties can easily be computed. Say, for example that two MSS:s are found that are sensitive to faults $f_{1}$ and $f_{2}$ according to Figure 1 a. In a noisy and uncertain environment we have to

\begin{tabular}{c|cc} 
& $f_{1}$ & $f_{2}$ \\
\hline$c_{1}$ & 0 & $X$ \\
$c_{2}$ & $X$ & $X$
\end{tabular}

(a) Fault incidence matrix.

Fig. 1. Isolability analysis

threshold the residuals such that the probability for false-alarm is low. Thus, conclusions about faults can only be drawn when a residual is larger than its associated threshold. When residuals are below thresholds, no conclusion can be drawn in a sound way. This means that for example $f_{2}$ may invalidate consistency relations $c_{1}$ and $c_{2}$. Thus, a large enough fault $f_{2}$ will be isolated as $f_{2}$. However, no matter how large, a fault $f_{1}$ can not be separated from $f_{2}$. This is summarized in the fault isolability matrix in Figure $1 \mathrm{~b}$ where an $X$ in position $(i, j)$ means that fault $i$ can be interpreted as fault $j$, i.e. fault $i$ can not be isolated from fault $j$.

Finally, a brief note on how time differentiation in dynamic systems is handled here. There are at least three different ways to represent time differentiated variables:

(1) Extend the model with equations describing how, for example, $x(t)$ is related to $\dot{x}(t)$ through the differentiation operator. This means that relations on the form

$$
\dot{x}=\frac{d x}{d t}
$$

is added for each variable that appear differentiated in the original model (Staroswiecki et al., 2000).

(2) Consider $x$ and $\dot{x}$ to be separate variables and perform structural differentiation of the model (Krysander and Nyberg, 2002b).

(3) Consider $x$ and $\dot{x}$ to be structurally the same variable and treat dynamic equations in the same way as static equations.

All three are possible choices, but for the isolability analysis performed here we use the third approach. Of course, time differentiated variables need to be represented if, in a second step, unknown variables are eliminated to form a consistency relation or a residual generator. Either of the two first suggestions above is possible and they both have advan- tages and disadvantages. This topic is not pursued further here.

\section{ILLUSTRATION ON SMALL EXAMPLE}

In this section, the structural analysis outlined in Section 2 will be applied to a small example model. In particular it will be shown how fault models can be introduced in the structural model to increase the fault isolability performance of the diagnosis system.

Now, consider the following small first-order dynamics model

$$
\begin{gathered}
\dot{x}=-\left(\alpha+f_{1}\right) x+u \\
y=x+f_{2}
\end{gathered}
$$

with two known signals, the control signal $u$ and the measurement $y$. The model is subjected to two faults where $f_{1}$ represents a change in the known parameter $\alpha$ and $f_{2}$ a fault in the sensor. Assume that we want to design a residual sensitive to fault $f_{2}$ but not to fault $f_{1}$, i.e. we want to isolate fault $f_{2}$ from fault $f_{1}$. It is clear that, with the information given in (2), no such residual exists and further knowledge about the process is needed. Now, assume that we know that the fault $f_{1}$ is slowly varying or, for a given limited time window, approximately constant. This information can be stated as

$$
\dot{f}_{1}=0
$$

With this new information it is possible to design a residual such that any constant $f_{1}$ does not influence the residual, i.e. a residual generator exists that fulfills the design specification. This could for example be done by designing an observer, estimating both the state and the fault $f_{1}$ with $y-\hat{y}$ as a residual. Now follows a description on how this straightforward fact also can be seen using only structural knowledge.

Consider again model equation (2), where the fault $f_{1}$ is considered as an unknown to be decoupled, the structural model becomes

\begin{tabular}{c|cc|cc|c} 
& $x$ & $f_{1}$ & $u$ & $y$ & $f_{2}$ \\
\hline$e_{1}$ & $X$ & $X$ & $X$ & & \\
$e_{2}$ & $X$ & & & $X$ & $X$
\end{tabular}

Here it is evident that no structurally singular/over determined subsystem exists since the system has two unknowns and only two equations. Inclusion of the fault model (3) gives the structural representation

\begin{tabular}{c|cc|cc|c} 
& $x$ & $f_{1}$ & $u$ & $y$ & $f_{2}$ \\
\hline$e_{1}$ & $X$ & $X$ & $X$ & & \\
$e_{2}$ & $X$ & & & $X$ & $X$ \\
$e_{3}$ & & $X$ & & &
\end{tabular}

Now, equations $\left\{e_{1}, e_{2}, e_{3}\right\}$ form a minimal structurally singular set of equations. Since $f_{2}$ appears in the equations in this set, any consistency relation for this set of equations may be sensitive to a change in $f_{2}$.

Thus, the analysis above states that there exists a residual generator such that $f_{1}$ is decoupled and the 
residual is sensitive to the fault $f_{2}$. Note that this is only a structural result, the particular form of the underlying analytical expression involved might make transformations into a residual generator impossible. However such topics are of course beyond what is possible to analyze using only structural models.

\section{MODEL OF DAMADICS VALVE}

In Section 5, isolability analysis is performed on the DAMADICS valve. Here, a brief introduction of the model is given. A schematic figure of the valve is shown in Figure 2 and consists of three main components: the control valve, by-pass valve, and a springand-diaphragm pneumatic servo-motor to operate the valve plug. The figure also shows an internal control loop that is used to increase the accuracy of the valve plug positioning. Measured variables of

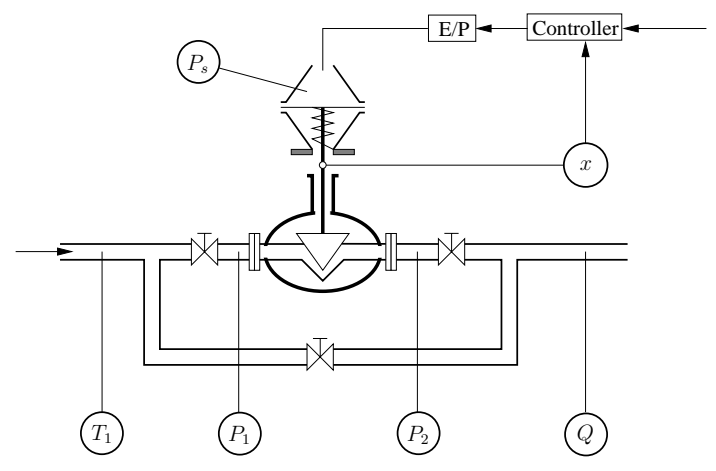

Fig. 2. Schematic figure of the DAMADICS valve

the valve, indicated by circles, are the valve plug position $x$, the fluid flow $Q$, fluid temperature $T_{1}$, up- and downstream pressure of the valve $P_{1}, P_{2}$, and the transducer chamber pressure $P_{s}$.

Details of this model is not included in this presentation, only the structure of the model is described. The presentation also shows the level of knowledge needed to make the analysis described and performed in Section 5. Readers interested in details of this model are referred to (WUT, 2002; Bartys, 2002; Bartys and Syfert, 2002) and the references therein.

\subsection{Spring-and-diaphragm pneumatic servo-motor}

This component consists of an electro-pneumatic transducer providing linear motion to the valveplug. Thus, the equations in this component describe the dynamics of the valve plug and the transducer chamber pressure $P_{s}$, which provides the main driving force of the plug.

The relative valve position $x$ is a dynamic function of the pressure in the chamber and the opposing force $F_{v c}$, the vena-contracta force, i.e.

$$
x=f\left(P_{s}, F_{v c}\right)
$$

This equation includes a model of the spring in the transducer and also friction components in the driving force. The pressure in the transducer chamber is a dynamic equation depending on the valve plug position (since this determines the effective volume of the chamber) and the net mass-flow of air $Q_{c}$ into the chamber. The inlet flow is a, also dynamic, function of the valve plug position controller $C V I$ output and the chamber pressure. Thus, these models can be summarized as

$$
\begin{aligned}
P_{s} & =f\left(x, Q_{c}\right) \\
Q_{c} & =f\left(P_{s}, C V I\right)
\end{aligned}
$$

\subsection{Control and bypass valve}

The valve equation describes the flow past the valve, $Q_{v}$, and the vena-contracta force. Both these entities are functions of the pressure upstream, $P_{1}$, and downstream, $P_{2}$, of the valve, the fluid temperature $T_{1}$ and the valve-plug position.

$$
\begin{aligned}
Q_{v} & =f\left(x, P_{1}, P_{2}, T_{1}\right) \\
F_{v c} & =f\left(x, P_{1}, P_{2}, T_{1}\right)
\end{aligned}
$$

The bypass valve is manually operated and only used when the flow past the control valve becomes choked. The flow past the bypass valve $Q_{v 3}$ obeys similar relations as the flow past the control valve.

$$
Q_{v 3}=f\left(x_{3}, P_{1}, P_{2}\right)
$$

where $x_{3}$ is the position of the manually operated by-pass valve.

\subsection{Fault modeling}

In (Bartys and Syfert, 2002), detailed fault models are described for 19 faults acting on the valve and its components. Typical faults in the valve are valve clogging, leakages, sensor faults, and different fault acting on the dynamics of the servo. In total, the control valve, the servo motor, and the positioner has 7,4 , and 4 modeled faults respectively. In addition 4 general/external faults are modeled. The faults are denoted $f_{1}, \ldots, f_{19}$ according to the order above.

No descriptions of the faults are included here and the reader is referred to (Bartys and Syfert, 2002) for details. In the analysis done in Section 5, we focus on 14 out of the 19 faults $^{2}$.

\subsection{Valve model summary}

The number of model equations of course depends on what form and how many intermediate variables that are used when forming the model. Here, when the model equations were collected, the result of the modeling is 19 equations out of which 3 are dynamic. The variables in the equations include 15 unknown variables, 14 faults, and 9 known signals. The 9 known signals consists of 6 sensor signals, the valveplug position controller reference value and output, and the position of the by-pass valve. The structure of the model is shown in Table 1.

2 Using the notation in (Bartys and Syfert, 2002), faults $f_{2}, f_{3}, f_{6}, f_{15}$, and $f_{17}$ are not considered here. 


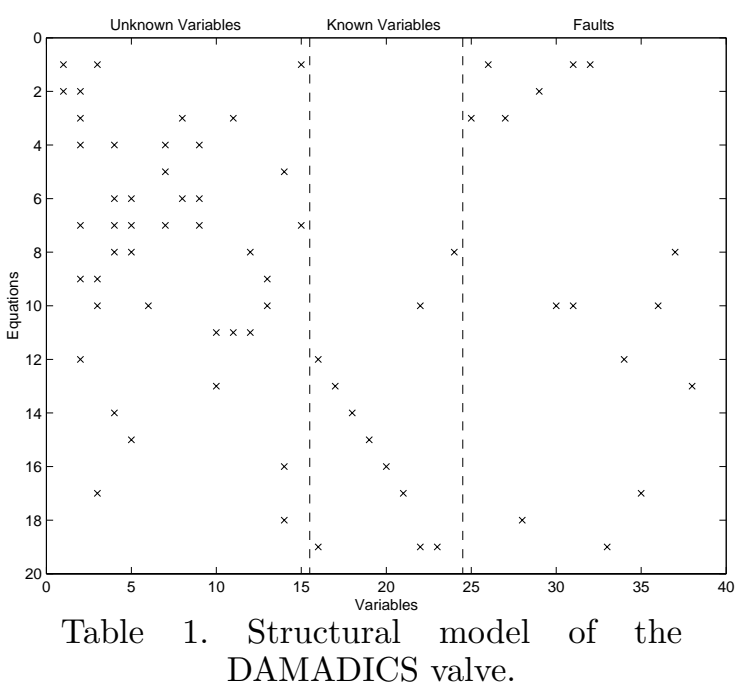

5. VALVE MODEL ANALYSIS

This section will describe a structural fault isolability analysis of the valve model and also find where additional modeling effort is needed to increase isolability properties.

\subsection{No decoupling of faults}

First, only knowledge on which faults that appear in which equations is used in the analysis. This is only a first step, if isolability properties is not satisfactorily, the residuals can be structured, i.e. faults can be decoupled. Decoupling of faults is further pursued in Section 5.2 and Section 5.3.

First, all MSS sets are computed based on the structural model of the valve. When computing the MSS sets, no faults are considered as unknown disturbances to be decoupled and all differentiated variables are considered structurally equivalent to its non-differentiated version. This results in $15 \mathrm{MSS}$ sets and for each MSS set, the fault sensitivity is computed. To visualize fault isolability properties of the computed MSS sets, a fault isolability analysis matrix is computed and shown in Table 2. To make

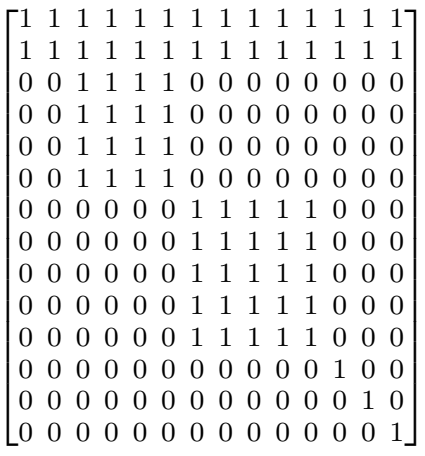

Table 2. Fault isolability analysis matrix with fault variable order $f_{16}, f_{9}, f_{19}, f_{18}$, $f_{5}, f_{1}, f_{14}, f_{11}, f_{10}, f_{8}, f_{4}, f_{7}, f_{12}, f_{13}$.

the analysis more clear, a Dulmage-Mendelsohn permutation is used to transform the fault matrix into an upper block-triangular form where isolability properties are clearly visible. Dulmage-Mendelsohn permutations only make sense in this case if row and column permutations are equal. This is not true in the general case, but due to the fact that the fault isolability matrix always has a non-zero diagonal, the row and column permutations are equal.

The fault sensitivity analysis first shows that faults $f_{16}$ and $f_{9}$ is not detectable in any MSS, i.e. we need additional sensors to be able to detect these two faults. In the fault isolability matrix this implies that the first two rows, corresponding to $f_{16}$ and $f_{9}$, have 1 in all positions. From the fault matrix we can draw additional conclusions that without any fault decoupling:

(1) The last three rows of the fault matrix show how faults $f_{7}$ (evaporation in the controlvalve), $f_{12}$ (fault in the electro-pneumatic transducer), and $f_{13}$ (fault in the valve-plug displacement sensor) are isolable from all other faults without any further need of fault modeling or fault decoupling.

(2) The two blocks of 4 and 5 faults respectively show groups of faults that are isolable from each other but where individual faults within each group is not isolable from the other faults in the group.

Further isolability analysis is concentrated on the second block of faults

\begin{tabular}{l|l} 
Fault & Description \\
\hline$f_{4}$ & Increase of valve or bushing friction \\
$f_{8}$ & Twisted servo-motor piston rod \\
$f_{10}$ & Servo-motor diaphragm perforation \\
$f_{11}$ & Servo-motor spring fault \\
$f_{14}$ & Pressure-sensor fault
\end{tabular}

\subsection{Decoupling of faults}

The next step in trying to increase isolability performance is to try and decouple faults in a group of un-isolable faults. If decoupling of one fault in the group of faults is possible without losing fault sensitivity to other faults in the group, isolability performance is increased. To analyze this, one fault at a time in the set $\left\{f_{4}, f_{8}, f_{10}, f_{11}, f_{14}\right\}$ is decoupled and the same analysis as in the previous section is performed. Performing these operations reveals that, in this case, the structure of fault influence is such that decoupling of the faults does not provide any additional isolability properties. The reason for this is that when decoupling one of the faults, any detectability of the other faults also vanishes and therefore also the possibility to isolate these faults from each other.

\subsection{Decoupling of fault models with additional fault models}

When decoupling of the faults, in a way outlined in the previous section, is not sufficient to achieve desired properties, additional information is needed. 
One such way is of course to include additional sensors. If this is not possible, further modeling of the faults is a solution. Assume that, by engineering knowledge, such additional knowledge about the faults are obtained. In general this is an equation on the form

$$
g(y, x, f)=0
$$

For example, if we know that the faults are slowly varying or approximately constant, relations on the form

$$
\dot{f}=0
$$

is added to the model equations. If the introduced fault model involves signals besides the fault signal, just introduction of the fault model might increase isolability performance. However, if the fault model is on the form (4) where only the fault signal is involved, additional decoupling is needed.

Here, model constraints on the form (4) is introduced. Performing the same decoupling procedure as in Section 5.2 a fault isolability matrix as in Table 3 is obtained. The conclusion from this is that with the

$$
\left[\begin{array}{llllllllllllll}
1 & 1 & 1 & 1 & 1 & 1 & 1 & 1 & 1 & 1 & 1 & 1 & 1 & 1 \\
1 & 1 & 1 & 1 & 1 & 1 & 1 & 1 & 1 & 1 & 1 & 1 & 1 & 1 \\
0 & 0 & 1 & 1 & 1 & 1 & 0 & 0 & 0 & 0 & 0 & 0 & 0 & 0 \\
0 & 0 & 1 & 1 & 1 & 1 & 0 & 0 & 0 & 0 & 0 & 0 & 0 & 0 \\
0 & 0 & 1 & 1 & 1 & 1 & 0 & 0 & 0 & 0 & 0 & 0 & 0 & 0 \\
0 & 0 & 1 & 1 & 1 & 1 & 0 & 0 & 0 & 0 & 0 & 0 & 0 & 0 \\
0 & 0 & 0 & 0 & 0 & 0 & \mathbf{1} & 0 & 0 & 0 & 0 & 0 & 0 & 0 \\
0 & 0 & 0 & 0 & 0 & 0 & 0 & \mathbf{1} & 0 & 0 & 0 & 0 & 0 & 0 \\
0 & 0 & 0 & 0 & 0 & 0 & 0 & 0 & 1 & 0 & 0 & 0 & 0 & 0 \\
0 & 0 & 0 & 0 & 0 & 0 & 0 & 0 & 0 & \mathbf{1} & 0 & 0 & 0 & 0 \\
0 & 0 & 0 & 0 & 0 & 0 & 0 & 0 & 0 & 0 & 1 & 0 & 0 & 0 \\
0 & 0 & 0 & 0 & 0 & 0 & 0 & 0 & 0 & 0 & 0 & 1 & 0 & 0 \\
0 & 0 & 0 & 0 & 0 & 0 & 0 & 0 & 0 & 0 & 0 & 0 & 1 & 0 \\
0 & 0 & 0 & 0 & 0 & 0 & 0 & 0 & 0 & 0 & 0 & 0 & 0 & 1
\end{array}\right]
$$

Table 3. Fault isolability analysis matrix after fault models are introduced. The variable order is the same as in Table 2.

additional fault models of faults $f_{4}, f_{8}, f_{10}, f_{11}$, and $f_{14}$, the structural analysis gives that it is possible to isolate the faults from each other.

\section{CONCLUSIONS}

This paper is concerned with structural isolability analysis of the DAMADICS valve model. It is shown how structural analysis can be used to assess isolability properties of a model. Since detailed knowledge about faulty systems is difficult to develop, it is essential to minimize this task. First, only information on which equations in the model that is influenced by the faults are provided. In Section 2 it is shown how structural analysis then provides information on which faults that need further modeling if the isolability objectives are to be met. It is also shown how additional fault models can be directly incorporated in the structural isolability analysis to ensure that the isolability objectives are met. The procedure is demonstrated in the DAMADICS valve model in Section 5 where it was concluded that 2 of the faults were not detectable with the set of sensors mounted on the valve. Three of the faults were uniquely isolable and the rest of the faults were grouped into two sets of faults, isolable from each other but not from faults within each group. For faults in these two groups, it is shown how additional fault models increases isolability performance of the model.

\section{ACKNOWLEDGMENT}

The authors acknowledge funding support under the EC RTN contract (RTN-1999-00392) DAMADICS. Thanks are expressed to the management and staff of the Lublin sugar factory, Cukrownia Lublin SA, Poland for their collaboration and provision of manpower and access to their sugar plant.

\section{REFERENCES}

M. Bartys. Specification of actuators intended to use for benchmark definition, v. 1.02. http://diag.mchtr.pw.edu.pl/damadics/, 2002.

M. Bartys and M. Syfert. Using damadics actuator benchmark library (dablib). Final, v. 1.21, 2002.

A.L. Gehin, M. Assas, and M. Staroswiecki. Structural analysis of system reconfigurability. In Proc. of IFAC Safeprocess'2000, volume 1, pages 292297, 2000.

M. Krysander and M. Nyberg. Structural analysis for fault diagnosis of DAE systems utilizing graph theory and MSS sets. Technical Report LiTH-R-2410, Department of Electrical Engineering, Linköping University, SE-581 83 Linköping, Sweden, 2002a.

M. Krysander and M. Nyberg. Structural analysis utilizing mss sets with application to a paper plant. Proc. of the Thirteenth International Workshop on Principles of Diagnosis, Semmering, Austria, 2002b.

B. Pulido and C. Alonso. An alternative approach to dependency-recording engines in consistencybased diagnosis. In Lecture Notes in Artificial Intelligence, volume 1904, pages 111-121. Artificial Intelligence: Methodology, Systems, and Applications. 9th International Conference, AIMSA 2000., Springer-Verlag, Berlin, Germany, 2000.

M. Staroswiecki, M. Cassar, and J.PH. Declerck. A structural framework for the design of FDI in large scale industrial plants. In Issues of fault diagnosis for Dynamic Systems ed. R. Patton, P. Frank, and R. Clark. Springer Verlag, 2000.

L. Travé-Massuyès, T. Escobet, and R. Milne. Model-based diagnosability and sensor placement. application to a frame 6 gas turbine subsystem. In D. T. Dupré S. Mcilraith, editor, DX01 twelfth international workshop on principals of diagnosis, pages 205-212, 2001

WUT. Benchmark definition, ver. 1.0. Benchmark for the DAMADICS valve, http://diag.mchtr.pw.edu.pl/damadics/, 2002. 